\title{
Prévalence des maladies virales du piment (Capsicum spp.) et perception des producteurs dans la commune de Malanville au Nord-Bénin
}

\author{
Fortuné BIAO ${ }^{1 *}$, Léonard AFOUDA ${ }^{1}$ et Daouda $\mathrm{KONE}^{2}$ \\ ${ }^{1}$ Laboratoire de Phytotechnie, d'Amélioration et de Protection des Plantes, Faculté d'Agronomie, Université \\ de Parakou, 02 B.P., 1003 Parakou, Bénin. \\ ${ }^{2}$ Laboratoire de Physiologie Végétale, UFR Biosciences, Université Félix Houphouët-Boigny, Abidjan 22 BP \\ 582, Côte d'Ivoire. \\ *Auteur correspondant, E-mail : fortunebiao@yahoo.fr / Tél :0022966945602.
}

\section{RESUME}

Au Bénin, le piment constitue l'un des condiments les plus demandés dans l'alimentation humaine. Cependant, sa production est confrontée à d'énormes contraintes dont les maladies virales. Pour lever ces contraintes qui contribuent à la baisse de rendement du piment, les maraîchers font recours aux pesticides chimiques aux conséquences néfastes pour les humains et pour l'environnement. La présente étude vise à identifier les maladies virales inféodées au piment à Malanville et à déterminer la perception des producteurs par rapport à ces maladies, afin de proposer des méthodes de lutte intégrées. Ainsi, une enquête et une prospection des champs ont été conduites à Malanville. Cent trente (130) échantillons de feuilles ont été collectés. La plus haute prévalence virale a été notée à Toumboutou (35\%). Les résultats des analyses sérologiques ont indiqué la présence du virus de la mosaïque du concombre (CMV) à 8,46\% et celui de la panachure du piment (PVMV) à $16.15 \%$ du taux d'infection sur 5 virus ciblés. Quatre-vingt-dix-neuf pourcent des marâichers affirment utiliser les insecticides chimiques pour lutter contre la présence de pucerons, mouches blanches et de thrips dans leur champ. La connaissance de la perception des maraîchers sur les maladies virales du piment et l'identification de ces virus sont importantes pour envisager des méthodes de lutte appropriées.

() 2019 International Formulae Group. All rights reserved

Mots clés: Piment, virus, pesticide, Malanville, Bénin.

\section{Prevalence of viral diseases of pepper (Capsicum spp.) and perception of producers in the district of Malanville in Northern Benin.}

\begin{abstract}
In Benin, pepper is one of the most popular condiments in the human diet. But, its production is confronted to enormous abiotic but also biotic constraints especially related to viral
\end{abstract}


diseases. To control these constraints contributing to pepper yield decrease, the producers often use chemical pesticides which are harmful to both humans and the environment. This study aims to identify the viral diseases that infest pepper cultures in Malanville and to determine the perception of producers related to these diseases in order to propose sustainable control methods. Thus, a survey was conducted in Malanville. 130 leaves samples were collected. The viral prevalence is highest in Toumboutou (35\%). Among the five viruses targeted during the survey, only PVMV $(16.15 \%)$ and CMV $(8.46 \%)$ were detected in the samples. To cope with the increasing prevalence of insects such as aphids, white flies and thrips and diseases, $99 \%$ of pepper producers claim to use chemical pesticides mainly made for cotton. The knowledge of truck farmers perceptions on pepper viral diseases and viruses identification make possible to look for appropriate control methods.

() 2019 International Formulae Group. All rights reserved.

Keywords: Pepper, virus, pesticide, Malanville, Benin.

\section{INTRODUCTION}

Parmi les cultures maraîchères, le piment (Capsicum spp., Solanaceae) occupe une place importante. En effet, c'est un légume-fruit cultivé de par le monde et dont l'importance dans l'alimentation humaine est capitale (Dias et al., 2013). Originaire d'Amérique du Sud et Centrale, il a été disséminé en Europe, en Afrique et en Asie (Menichini et al., 2009; Zimmer et al., 2012). Le piment est classé parmi les premiers épices alimentaires et fait partie des 40 espèces de légumes les plus produites dans le monde entier (Lebeau, 2010).

$\mathrm{Au}$ Bénin, après la tomate, le piment constitue le condiment le plus demandé dans les milieux urbains pour la consommation à cause de sa saveur piquante (Gnimadi, 2008). Il est cultivé aussi bien en saison de pluie qu'en contre-saison. Vu l'importance de la filière piment au Bénin, elle a été retenue dans le Plan Stratégique de Relance du Secteur Agricole, adopté en octobre 2011 par le gouvernement béninois, en tant que filière à promouvoir (MAEP, 2011).

Cependant, malgré les efforts du gouvernement Béninois visant à améliorer sa production, son rendement de 2,71 tonnes/hectare contre un rendement mondial de 16,68 tonnes/hectare reste très faible FAO (2016). En effet, sa production fait face à de nombreuses contraintes, aussi bien abiotiques que biotiques. Parmi les facteurs abiotiques, on peut citer les effets des changements climatiques avec des perturbations qui impactent négativement les rendements agricoles à travers la dégradation et la perte de fertilité des sols, la prolifération des insectes ravageurs et des maladies (Katé et al., 2014). En ce qui concerne les différentes contraintes biotiques, celles causées par les phytovirus transmissibles par les insectes vecteurs constituent un problème économique et agricole majeur (Afouda et al.,2013).

$\mathrm{Au}$ total, 68 virus ont été décrits sur le piment dans le monde (Pernezny et al., 2003). En Tunisie, en pleine saison, la culture de piment est fortement endommagée par le virus $\mathrm{Y}$ de la pomme de terre (PVY) et le virus de la mosaïque du concombre (CMV), causant plus de $50 \%$ des pertes de la production potentielle des variétés de piment cultivées (Mnari-Hattab et Ezzaier, 2006).

Au Bénin, très peu de travaux ont été conduits sur les contraintes phytosanitaires du piment. Les travaux de Afouda et al., (2013; 2017), ont révélé sur le piment la présence de certains virus tels que le virus $\mathrm{Y}$ de la pomme de terre (PVY), le virus de la panachure du piment (PVMV), le virus de la mosaïque du concombre (CMV), le Virus des feuilles jaunes en cuillère de la tomate (TYLCV), le virus de la mosaïque du tabac (TMV) et le virus de la mosaïque de la tomate (ToMV). 
Aussi, Tossounon et Onzo (2015) ont rapporté la présence des arthropodes vecteurs de maladies tels que des acariens et des insectes, notamment les thrips (Frankliniella schultzei Trybom), les mouches blanches (Bemisia tabaci Gennadius) et majoritairement les pucerons (Aphis gossypii Glover).

Pour faire face à cette pression due aux insectes nuisibles avec les maladies qu'ils transmettent, les maraîchers font recours et de façon abusive à l'utilisation des pesticides chimiques hautement toxiques et polluants (Mondedji et al.,2015). Les conséquences en sont l'intoxication des maraîchers et des consommateurs, la pollution de l'environnement et la sélection de souches d'insectes résistantes aux pesticides (Gnago et al., 2010 ; Son et al., 2017). Des nombreuses études scientifiques existantes sur le piment au Bénin, peu d'entre-elles ont abordé la perception des maraîchers de Malanville sur les thèmes relatifs aux insectes nuisibles, aux maladies virales du piment et leur transmission et aux différentes méthodes de lutte. La présente étude vise d'une part à comprendre la perception des maraîchers de la commune de Malanville sur les thèmes cidessus cités et d'autre part, l'actualisation de l'état des connaissances des maladies virales du piment dans la commune de Malanville afin de proposer des méthodes adéquates de lutte.

\section{MATERIEL ET METHODES Milieu d'étude}

L'enquête et la prospection ont été réalisées dans la commune de Malanville, située à l'extrême Nord du Bénin dans le département de l'Alibori. Elle s'étend entre $11,5^{\circ} 00^{\prime}$ 'et $12^{\circ} 00^{\prime}$ de latitude Nord et entre $2^{\circ} 45^{\prime}$ et $3^{\circ} 40^{\prime}$ de longitude Est, et à une altitude moyenne de $200 \mathrm{~m}$ au-dessus du niveau de la mer. Dans cette commune, le climat est de type soudano-sahélien, marqué par une saison sèche de Novembre à Avril. Cette zone est caractérisée par une forte production des cultures maraîchères dont la culture du piment (Dossou et al, 2006). Ce qui a motivé le choix de cette commune pour notre étude.

\section{Identification des maladies virales du piment à Malanville}

La prospection a pris en compte les cinq arrondissements de la commune de Malanville. Le nombre d'échantillons et de sites est fonction de la taille de production de piment dans chaque arrondissement. $\mathrm{La}$ collecte des échantillons sur les sites a été effectuée suivant la méthode des diagonales. Les jeunes feuilles apicales ont été échantillonnées sur cinq (5) plants de piment choisis par site pour la détermination de l'incidence des maladies virales. Ces feuilles ont été choisies sur la base d'observation visuelle des symptômes de maladies virales (rabougrissement, retard de croissance, mosaïque, taches nécrotiques, feuilles recroquevillées ou en cuillère) et de la présence d'insectes vecteurs. Les échantillons étiquetés ont été conservés dans de la glace puis transférés au laboratoire où ils ont été conservés à $-20{ }^{\circ} \mathrm{C}$ en attendant les tests d'identification ELISA. Un total de 130 échantillons a été prélevé sur 26 sites.

$\mathrm{Au}$ laboratoire, chaque échantillon de feuille de piment a été broyé et homogénéisé avec le tampon d'extraction spécifique à chaque format du test ELISA à raison de $0,1 \mathrm{~g}$ de feuille pour $2 \mathrm{~mL}$ de tampon d'extraction; soit une dilution de $1 / 20$ (poids/volume). L'identification d'antigène viral dans chaque échantillon a été effectuée en utilisant cinq (05) anticorps dirigés respectivement contre le $\mathrm{CMV}$, le PVMV, le PVY, le TYLCV et le TSWV à travers trois formats du test immunologique ELISA comme décrit par Afouda et al.(2013) et conformément aux recommandations du fabriquant. Il s'agit de DAS-ELISA pour le PVMV et le CMV, d'ACP-ELISA pour le PVY et du TAS-ELISA pour le TYLCV et le TSWV. Ces anticorps fournis par le département de virologie de la Deutsche Sammlung von Mikroorganismen und Zellkulturen (DSMZ) sont présentés dans le Tableau 1. 
Tableau 1: Les anticorps utilisés.

\begin{tabular}{c|ccc}
\hline \multicolumn{1}{c}{ Virus } & Code des anticorps & Dilutions recommandées & Format du test \\
\hline$C M V$ & AS-0929 & $1: 1000$ & DAS-ELISA \\
\hline$P V M V$ & AS-0123 & $1: 1000$ & DAS-ELISA \\
\hline$P V Y$ & AS-0343 & $1: 1000$ & ACP- ELISA \\
\hline$T Y L C V$ & AS-0588 & $1: 1000$ & TAS-ELISA \\
\hline$T S W V$ & AS-0105 & $1: 1000$ & TAS-ELISA \\
\hline
\end{tabular}

L'évaluation des résultats sur les plaques a été faite au lecteur de plaques ELISA Biotek (ELX800) à une longueur d'onde de $405 \mathrm{~nm}$. La lecture a été faite 1 heure après incubation des plaques avec le substrat P-nitrophenyl Phosphate à $1 \mathrm{mg} \cdot \mathrm{ml}^{-1}$. Les échantillons positifs ont été déterminés à partir des valeurs du témoin négatif constitué d'un échantillon de feuille non virosé. Sont considérés comme positifs les échantillons dont les valeurs d'absorbance sont deux fois supérieures ou égales à la moyenne de celles du témoin négatif (Ryu et al., 2009).

\section{Méthodes d'enquête}

Une enquête randomisée par entretien semi-directif et par observation participante a été conduite dans tous les arrondissements de la commune de Malanville du 25 septembre 2017 au 6 janvier 2018. L'enquête s'est déroulée à l'aide d'un Smartphone muni du logiciel KoBoCollect dans lequel les questionnaires ont été précédemment intégrés. Les entretiens avec les producteurs de piment ont porté principalement sur les principaux problèmes phytosanitaires et les méthodes de lutte utilisées.

Les réponses aux questionnaires ont été envoyées sur la plateforme KoBoToolbox. Les données ont ensuite été exportées dans le logiciel Excel puis soumises à l'analyse statistique à l'aide du logiciel $\mathrm{R}$ version 3.1.2. Parallèlement à l'enquête, une prospection a été réalisée et les feuilles de piment ont été collectées pour identifier les maladies virales du piment à Malanville.

\section{Analyse statistique}

Les fréquences et les moyennes relatives aux données d'enquête ont été déterminées à l'aide $\mathrm{du}$ logiciel $\mathrm{R}$ version 3.4.1 ( $\mathrm{R}$ Core Team 2017). Les modèles linéaires généralisés avec la distribution de la famille binomiale ont été utilisés pour tester l'effet des arrondissements sur l'incidence de chacun des virus identifiés au cours de la prospection (R Core Team 2017).

\section{RESULTATS}

\section{Prévalence des maladies virales}

Sur les 130 échantillons de piment collectés et testés, 32 échantillons (soit $24,61 \%$ ) ont réagi positivement aux anticorps pour deux différents virus que sont: le CMV avec 11 échantillons positifs (soit 8,46\%) et le PVMV avec 21 échantillons positifs (soit $16,15 \%)$. Aucun échantillon n'a été testé positif pour le PVY, le TYLCV et le TSWV. Deux échantillons (soit 1,53\%) présentaient un cas d'infection double par le CMV et le 
PVMV. La prévalence du PVMV est pratiquement le double de celle du CMV. La prévalence virale par arrondissement est indiquée dans le Tableau 2.

La prévalence cumulée du CMV et du PVMV n'est pas significativement différente d'un arrondissement à l'autre $(\mathrm{p}>0,05)$. Toutefois, cette prévalence est relativement plus élevée dans certains arrondissements dont Toumboutou (35\%) et faible dans d'autres dont Garou (12,25\%). Par rapport à la prévalence de chaque virus pris séparément, seule la prévalence du PVMV diffère significativement entre les arrondissements $(p<0,05)$. Elle est plus élevée à Malanville $(31,42 \%)$ et plus faible à Madécali (5\%). Par contre, la prévalence du CMV est plus élevée à Guéné (20\%) et moins élevée à Malanville (2,85\%). Les virus PVY, TYLCV et TSWV n'ont été détectés dans aucun des 5 arrondissements considérés, Tableaux 2 et 3 .

\section{Perception des maraîchers de Malanville} sur les maladies virales du piment Capsicum spp. et les différentes méthodes de lutte appliquées

\section{Importance du piment dans la commune de} Malanville

Selon les maraîchers enquêtés, le piment est majoritairement repiqué dans la commune de Malanville de Juin à Septembre, avec un pic de repiquage en Août. Cette enquête nous révèle que $67,67 \%$ des maraîchers préfèrent cultiver le piment vert (Capsicum chinense Jacq), appelé localement en dendi Gbatakin contre $32,33 \%$ qui préfèrent le piment long (Capsicum anпиит L.), localement appelé en dendi Bongosigui. Tous les enquêtés affirment que les semences de piment proviennent des cultures précédentes. La superficie moyenne emblavée par maraîcher est d'environ 0,75 ha. La culture du piment est majoritairement effectuée en monoculture mais, parallèlement au champ de piment, $79,79 \%$ des enquêtés cultivent de l'oignon, de la tomate, du gombo, du mil, du sorgho et du riz.

\section{Importance des insectes rencontrés en culture de piment}

Tous les maraîchers enquêtés (100\%) affirment rencontrer plus d'insectes ravageurs tels que les pucerons, les mouches blanches, les thrips dans leur champ de piment en saison sèche qu'en saison pluvieuse. Selon eux, les pucerons sont les plus dévastateurs. Ils sont beaucoup plus fréquents aux stades de fructification $(59,59 \%)$, de floraison $(20,20 \%)$, de forte croissance végétative $(16,16 \%)$, de pépinière $(3,03 \%)$ et de graines $(1,02 \%)$. Les dommages occasionnés par ces insectes sont: la perforation des feuilles, la perte de rendement, la perte de qualités des fruits qui deviennent collants et de petite taille, la mort des plantes. Tous les enquêtés affirment que les insectes et les maladies sont plus fréquents actuellement et constituent le principal frein au développement de la filière piment à Malanville.

Perception des maraîchers sur les maladies virales du piment et méthodes de lutte

Tous les maraîchers enquêtés affirment ne pas pouvoir différencier les maladies virales des maladies bactériennes et fongiques. Ainsi, 90\% d'entre-eux n'ont jamais entendu parler de phytovirus et de leur mode de transmission. Par contre, 10\% affirment avoir entendu parler de phytovirus mais ne connaissent pas leur mode de transmission et ne peuvent non plus les différencier des autres maladies inféodées aux plants de piment. Après une brève présentation des symptômes aux paysans, tous affirment rencontrer ces symptômes dans leur 
champ de piment. Pour lutter contre les maladies virales du piment, $3,02 \%$ des maraichers utilisent des variétés de piment améliorées obtenues auprès du Secteur Communal du Développement Agricole (SCDA) de Malanville. Quatre-vingt-dix-neuf pour cent (99\%) des maraîchers utilisent les pesticides chimiques pour lutter contre les insectes ravageurs et les différentes maladies du piment. Parmi les pesticides utilisés on retrouve l'insecticide systémique localement appelé Pia-Pia (Dichlorvos ou DDVP), extrêmement toxique appartenant à la famille des Organophosphorés (83,50\%), l'insecticide systémique localement appelé "Premier" largement utilisé dans la culture du coton, du nom commercial Thalis 112 EC ou SIBEMAC 112 EC (Emamectine benzoate à $48 \mathrm{~g} / \mathrm{L}$ et l'acétamipride à $64 \mathrm{~g} / \mathrm{L}),(29.47 \%)$. Le Rambo "insecte power" (perméthrine) de la famille des pyréthrinoïdes et le PACHA (Acétamipride 45g/L, lambdacyhalothrine) sont également utilisés respectivement par $21,05 \%$ et $6,32 \%$ des maraîchers. Ces insecticides sont majoritairement achetés sur le marché local sans aucune garantie d'efficacité.

Quant aux méthodes de lutte alternatives, cette enquête révèle que 64,85\% des maraîchers ne savent pas qu'on pourrait utiliser des extraits de plantes pour lutter contre les insectes vecteurs de maladies virales. Par contre, $35,15 \%$ savent qu'il existe des plantes à effets insecticides qui pourraient être utilisées. Cependant, ils ne les pratiquent pas dans leur champ car, disent-ils, la préparation demande assez de temps. Parmi ceux qui connaissent la vertu insecticide des plantes, 94,29\% ont cité le Neem et 5,71\% l'Hyptis. Les cultures pièges avec sorgho, mil, maïs etc, mises en place comme cultures de bordure sont, par contre peu pratiquées par les maraîchers $(20,20 \%)$.

\section{DISCUSSION}

\section{Prévalence des maladies virales}

Sur les 130 échantillons prélevés, seulement 32 échantillons se sont révélés positifs au test ELISA. Le statut négatif des autres échantillons malgré les symptômes observés lors du prélèvement pourrait être dû à d'autres virus que notre étude n'a pas pris en compte. Cela pourrait également être dû aux problèmes d'ordre abiotique ou à une simple infestation des ravageurs qui se seraient alimentés sans transmettre de virus. Ainsi, les symptômes ne sont pas forcément causés par les virus recherchés, ils pourraient dériver d'un autre pathogène non identifié ou ravageur (autres virus, champignons, bactéries, insectes, acariens, etc). Après les analyses, les échantillons révélés positifs sont infectés par deux virus que sont le PVMV avec une incidence de $16,15 \%$ et le CMV avec une incidence de $8,46 \%$. Ces virus font partie de ceux détectés par Afouda et al. (2013). Par contre le PVYn, TYLCV qui avaient été précédemment détectés par ces mêmes auteurs; n'ont pas été identifiés dans notre étude malgré la présence des mouches blanches vecteurs du TYLCV sur les plantes de piment lors de l'échantillonnage. Cela pourrait être dû à l'absence parmi ces mouches blanches de celles vectrices de ce virus. Nzi et al. (2010) ont effectué un lien entre le niveau de pullulation des mouches blanches et la période de semis de la tomate au centre de la Côte d'Ivoire. Il ressort de leur étude que lors des semis de saison des pluies, il n'y a aucune épidémie du TYLCV malgré la présence de la mouche blanche. Nos résultats sont conforment à cette observation car notre 
Tableau 2: Prévalence (\%) des virus par arrondissement dans la commune de Malanville / Prevalence (\%) of viruses by borough in Malanville.

\begin{tabular}{|c|c|c|c|c|c|c|c|c|}
\hline Arrondissements & $\begin{array}{l}\text { Nombre } \\
\text { d'échantillons }\end{array}$ & CMV & PVMV & PVYn & TYLCV & TSWV & CMV+PVMV & Total \\
\hline Garou & 40 & 5 & 7,5 & 0 & 0 & 0 & 0 & 12,5 \\
\hline Guéné & 15 & 20 & 13,33 & 0 & 0 & 0 & 0 & 33,3 \\
\hline Madécali & 20 & 10 & 5 & 0 & 0 & 0 & 0 & 15 \\
\hline Malanville & 35 & 2,85 & 31,42 & 0 & 0 & 0 & 1 & 34,29 \\
\hline Toumboutou & 20 & 15 & 20 & 0 & 0 & 0 & 1 & 35 \\
\hline Total & 130 & 11 & 21 & 0 & 0 & 0 & 2 & 24,6 \\
\hline
\end{tabular}

Tableau 3: Comparaison de la prévalence du PVMV et du CMV entre arrondissements de Malanville / Comparison of PVMV and CMV prevalence between Malanville's borough.

\begin{tabular}{|c|c|c|c|c|c|c|c|c|c|c|c|c|}
\hline \multicolumn{5}{|c|}{ PVMV } & \multicolumn{4}{|c|}{ CMV } & \multicolumn{3}{|c|}{ PVMV+CMV } & \multirow[b]{2}{*}{$\operatorname{Pr}(>|z|)$} \\
\hline $\begin{array}{l}\text { Sources } \\
\text { de variation }\end{array}$ & Estimate & Std.Error & $\begin{array}{c}z- \\
\text { value }\end{array}$ & $\overline{\operatorname{Pr}(>|\mathrm{z}|)}$ & Estimate & Std.Error & z-value & $\operatorname{Pr}(>|z|)$ & Estimate & Std.Error & $\begin{array}{c}z- \\
\text { value }\end{array}$ & \\
\hline (Intercept) & -2.5123 & 0.6003 & -4.185 & $\begin{array}{l}2.85 \mathrm{e}- \\
05 * * *\end{array}$ & -2.9444 & 0.7255 & -4.059 & $194 \mathrm{e}-05^{* * *}$ & -1.9459 & 0.4781 & -4.070 & $4.7 \mathrm{e}-05^{* * *}$ \\
\hline Guéné & 0.6405 & 0.9681 & 0.662 & 0.5082 & 1.5581 & 0.9711 & 1.605 & 0.109 & 1.2528 & 0.7270 & 1.723 & 0.0849 \\
\hline Madécali & -0.4321 & 1.1886 & -0.364 & 0.7162 & 0.7472 & 1.0401 & 0.718 & 0.473 & 0.2113 & 0.1879 & 0.268 & 0.7885 \\
\hline Malanville & 1.7321 & 0.7021 & 2.467 & $0.0136^{*}$ & -0.5819 & 1.2473 & -0.467 & 0.641 & 1.1658 & 0.6010 & 1.940 & 0.0524 \\
\hline Toumboutou & 1.1260 & 0.8203 & 1.373 & 0.1698 & 1.2098 & 0.9584 & 1.262 & 0.207 & 1.0986 & 0.6831 & 1.608 & 0.1078 \\
\hline
\end{tabular}

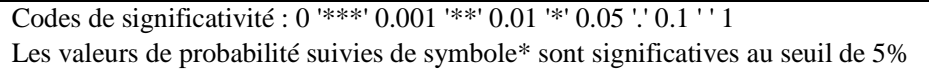


prospection a été effectuée dans le mois de Septembre qui était encore arrosé par les dernières pluies. Quant au TSWV, malgré la présence des thrips, il s'est révélé négatif sur tous les échantillons collectés. Ce résultat vient confirmer ceux obtenu par Afouda et al. (2013) dans le Nord Bénin. Par ailleurs, nos études révèlent que l'incidence du PVMV est supérieure à celle du CMV. Afouda et al. (2013, 2017) avaient remarqué la même tendance dans le Nord-Bénin et ensuite dans le Sud-Bénin.

Lors de la prospection, nous avons constaté que, d'un arrondissement à un autre, la production de piment n'est pas la même. En effet, certains arrondissements produisent plus de piment que d'autres. Par ailleurs, notre étude révèle que la prévalence des maladies virales est faible à Garou et à Madécali. Cette faible prévalence enregistrée dans ces deux arrondissements serait due au fait que dans ces régions, la culture de piment est beaucoup plus associée à celle de l'oignon qui possède des propriétés répulsives sur les insectes (Auger et al., 2013).

Par contre, à Malanville et à Toumboutou la culture de piment est beaucoup plus pratiquée en monoculture. $\mathrm{Ce}$ système cultural aurait favorisé le développement des insectes vecteurs de maladies virales dans ces deux arrondissements (James et al., 2010).

\section{Perception des maraîchers de Malanville} sur les maladies virales du piment Capsicum spp. et les différentes méthodes de lutte appliquées.

A Malanville, le piment vert (Gbatakin) est le plus cultivé. Ce choix des maraîchers serait dû à sa forte demande sur les marchés nationaux et sous régionaux. Le piment est majoritairement repiqué à Malanville au mois d'Août car, ce mois représente le mois le plus arrosé dans la commune. Aussi, les semis en fin Août permettent d'avoir des cultures de contre saison. Lesquelles sont plus rentables pour les maraîchers.

$\mathrm{Au}$ cours de cette enquête tous les maraîchers affirment rencontrer dans leurs champs les pucerons, les mouches blanches et les thrips. Cette affirmation corrobore les résultats obtenus par Tossounou et Onzo (2015) au cours de leur étude sur l'inventaire des acariens et insectes du piment vert à Kandi et à Malanville. Ils affirment rencontrer plus d'insectes en saison sèche, de Décembre à Mars.

Les maraîchers de Malanville ignorent le concept de maladies virales et de leur transmission. Par conséquent, ils ne différencient pas les maladies virales des autres pathologies. Cette ignorance des maraîchers pourrait entacher la qualité des traitements à travers l'usage abusif et inapproprié des insecticides (Son et al., 2017). La lutte contre les maladies des plantes de piment à travers l'usage des variétés améliorées est peu connue et peu utilisée par les maraîchers de Malanville. Cependant, les traitements phytosanitaires à base de pesticides chimiques de synthèse sont largement utilisés. Ceci a été également révélé chez les maraîchers au sud du Togo (Mondedji et al., 2016) et ceux des périmètres de Houéyiho et de Parakou qui affirment qu'il n'est pas possible de cultiver les légumes sans utiliser des insecticides (Akogbeto et al., 2005). Son et al. (2017) ont également observé une forte utilisation des pesticides chimiques par les producteurs de tomate au Burkina Faso. Il ressort de l'enquête que 
l'insecticide Pia-Pia dont la matière active est le Dichlovos ou DDVP est le plus utilisé par les maraîchers de Malanville, bien qu'il soit hautement toxique et retiré du marché (Aminou, 2016). La quasi-totalité des insecticides utilisés par les marâichers de Malanville sont des insecticides recommandés sur le coton. Or, l'usage des pesticides destinés aux cotonniers en maraîchage est interdit en raison de leur persistance sur les cultures et de leur forte toxicité (Schiffers et Mar, 2011). Le non-respect des recommandations liées à la date de récolte après traitement et l'usage abusif et répétitif des pesticides coton en maraîchage mettent en péril la santé des consommateurs et impactent négativement l'environnement (Agboyi et al., 2015) et les organismes aquatiques à travers la pollution des cours d'eau (Agbohessi et al., 2012). Cette mauvaise gestion des pesticides chimiques induit la résistance des insectes aux différents principes actifs et par conséquent l'émergence des différentes pathologies (Anstead et al., 2005). Face à ce phénomène, il urge de promouvoir la lutte intégrée incluant le développement des semences améliorées résistantes aux insectes et maladies ainsi que l'usage des biopesticides.

\section{Conclusion}

A l'issu de cette étude, il est important de retenir que les pucerons, les mouches blanches et les thrips sont les insectes les plus rencontrés dans la culture de piment à Malanville. Les virus PVMV et CMV ont été identifiés sur le piment. Le concept de maladie virale et de sa transmission est peu connu par les maraîchers. Ainsi donc, pour lutter contre ces maladies, ces derniers utilisent majoritairement les insecticides coton tels que le Pia-Pia (Dichlorvos) hautement toxique aussi bien pour les consommateurs que pour l'environnement. La connaissance de la perception des maraîchers de Malanville sur les maladies virales et sur les méthodes de lutte appliquées, de même que l'identification des principaux virus inféodés à la culture de piment, nous permettront de proposer les méthodes de luttes adéquates et efficaces contre les maladies virales du piment qui constituent un frein au développement de cette filière dans la commune de Malanville.

\section{CONFLIT D'INTERETS}

Les auteurs n'ont déclaré aucun conflit d'intérêt.

\section{REMERCIEMENTS:}

Ce travail a été financé par le Centre d'Excellence Africain en Changement Climatique, Biodiversité et Agriculture Durable et l'Union Economique et Monétaire Ouest Africaine. J'adresse mes sincères remerciements aux collègues des Universités de Parakou et de Félix Houphouët-BOIGNY qui ont contribué à l'amélioration de ce travail.

\section{REFERENCES}

Afouda L, Kone D, Zinsou V, Dossou L, Kenyon L, Winter S, Knierim D. 2017. Virus surveys of Capsicum spp. in the Republic of Benin reveal the prevalence of pepper vein yellows virus and the identification of a previously uncharacterised polerovirus species. Archives of Virology, 162: 1599-1607. DOI: https://doi.org/10.1007/s00705017-3274-8

Afouda L, Kotchofa R, Sare R, Zinsou V, Winter S. 2013. Occurrence and distribution of viruses infecting tomato 
and pepper in Alibori in northern Benin.

Phytoparasitica, 41: 271-276. DOI:

https://doi.org/10.1007/s12600-013-

0287-z

Agbohessi TP, Toko II, Kestemont P. 2012. État des lieux de la contamination des écosystèmes aquatiques par les pesticides organochlorés dans le Bassin cotonnier béninois. Cahiers Agricultures, 21: $46-56 . \quad$ DOI: https://doi.org/10.1684/agr.2012.0535

Agboyi LK, Djade KM, Ahadji-Dabla KM, Ketoh GK, Nuto Y, Glitho IA. 2015. Vegetable production in Togo and potential impact of pesticide use practices on the environment. International Journal of Biological and Chemical Sciences, 9(2): 723-736. DOI : http://dx.doi.org/10.4314/ijbcs.v9i2.13

Akogbeto M, Djouaka C, Noukpo H. 2005. Utilisation des insecticides agricoles au Bénin. Bulletin de la Société de Pathologie Exotique, 98(5): 400-405

Aminou S. 2016. Les produits de type «piapia » à base de la matière active Dichlorvos ou DDVP dont l'utilisation est à supprimer au Niger. Réseau National des Chambres d'Agriculture du Niger (RECA). 3 p.

Anstead JA, Williamson MS, Denholm I. 2005. Evidence for multiple origins of identical insecticide resistance mutations in the aphid Myzus persicae. Insect Biochemistry and Molecular Biology, 35: 249-256.

DOI: https://doi.org/10.1016/j.ibmb.2004.12.0 04

Auger J, Lecomte C, Thibout E. 2013. Les composés soufrés des Allium: leurs activités biologiques chez les insectes et leur production. Acta Botanica Gallica,
140: $\quad 157-168 . \quad$ DOI: https://doi.org/10.1080/12538078.1993.1 0515580

Dias GB, Gomes VM, Moraes TM, Zottich UP, Rabelo GR, Carvalho AO, Moulin M, Gonçalves LS, Rodrigues R, Da Cunha M. 2013. Characterization of Capsicum species using anatomical and molecular data. Genetics and Molecular Research, 12: 6488-6501. DOI: http://dx.doi.org/10.4238/2013.February. 28.29

Dossou J, Soulé I, Montcho M. 2006. Analyse économique de la production de purée de tomate à petite échelle au Bénin. Tropicultura, 24: 239-246.

$\begin{array}{ccr}\text { FAOSTAT. 2016. } & \text { FAOSTAT } & \text { (FAO } \\ \text { Statistics) } & \text { Available } & \text { at } \\ \text { http://faostat.fao.org. } & \text { Consulté } & \text { le } \\ \text { 01/01/2018 } & & \end{array}$

Gnago JA, Danho M, Agneroh TA, Fofana IK, Kohou AG. 2010. Efficacité des extraits de neem (Azadirachta indica) et de papayer (Carica papaya) dans la lutte contre les insectes ravageurs du gombo (Abelmoschus esculentus) et du chou (Brassica oleracea) en Côte d'Ivoire. International Journal of Biological and Chemical Sciences, 4: 953-966. DOI: http://dx.doi.org/10.4314/ijbcs.v4i4.6303 5

Gnimadi A. 2008. Etude pour l'identification des filières agroindustrielles prioritaires. Programme de Restructuration et de Mise à Niveau de l'Industrie des Etats membres de l'UEMOA (PRMN).118 p.

James B, Atcha-Ahowé C, Godonou I, Baimey H, Goergen G, Sikirou R, Toko M. 2010. Gestion Intégrée des Nuisibles en Production Maraîchère: Guide pour les Agents de Vulgarisation en Afrique 
de l'Ouest. Institut International d'Agriculture Tropicale (IITA): Ibadan, Nigeria; $\mathrm{p} 120$.

Katé S, Dagbenonbakin GD, Agbangba CE, de Souza JF, Kpagbin G, Azontondé A, Ogouwalé E, Tinté B, Sinsin B. 2014. Perceptions locales de la manifestation des changements climatiques et mesures d'adaptation dans la gestion de la fertilité des sols dans la Commune de Banikoara au Nord-Bénin. Journal of Applied Biosciences, 82: 7418-7435. DOI: http://dx.doi.org/10.4314/jab.v82i1.111

Lebeau A. 2010. Résistance de la tomate, l'aubergine et Piment à Ralstonia solanacearum: Interaction entre les géniteurs de Résistance et la diversité bactérienne, caractérisation et cartographie des facteurs génétiques impliqués chez l'aubergine. Thèse de Doctorat, Université de la Réunion, $178 \mathrm{p}$.

Menichini F, Tundis R, Bonesi M, Loizzo MR, Conforti F, Statti G, De Cindio B, Houghton PJ. 2009. The influence of fruit ripening on the phytochemical content and biological activity of Capsicum chinense Jacq. cv Habanero. Food Chemistry, 14: 553-560. DOI: https://doi.org/10.1016/j.foodchem.2008. 09.086

Ministère de l'Agriculture, de l'Elevage et de la Pèche. 2011. Plan Stratégique de Relance du Secteur Agricole (PSRSA). Consulté le 19/03/2018. http://extwprlegs1.fao.org/docs/pdf/ ben149176.pdf

Mnari-Hattab M, Ezzaier K. 2006. Biological, serological, and molecular characterization of Pepper mild mottle virus (PMMoV) in Tunisia. Tunisian Journal Plant Protection, 1:1-12.

Mondedji AD, Nyamador WS, Amevoin K, Adéoti R, Abbévi AG, Koffivi GK, Adolé GI. 2015. Analyse de quelques aspects du système de production légumière et perception des producteurs de l'utilisation d'extraits botaniques dans la gestion des insectes ravageurs des cultures maraîchères au Sud du Togo. International Journal of Biological and Chemical Sciences, 9(1): 98-107. DOI: http://dx.doi.org/10.4314/ijbcs.v9i1.10

Mondedji AD, Kasseney BD, Nyamador WS, Abbévi Abbey G, Amevoin K, Adéoti R, Ketoh KG, Glitho IA. 2016. Effets d'extrait hydroéthanolique de feuilles de neem (Azadirachta indica A. Juss) sur Plutella xylostella (Lepidoptera: Plutellidae) et Lipaphis erysimi (Hemiptera: Aphididae) dans la production du chou au Sud du Togo. International Journal of Biological and Chemical Sciences, 10: 1666-1677. DOI: http://dx.doi.org/10.4314/ijbcs.v8i5.30.

Nzi J, Kouamé C, N'guetta A, Fondio L, Djidji A, Sangare A. 2010. Evolution des populations de Bemisia tabaci Genn. selon les variétés de tomate (Solanum lycopersicum L.) au Centre de la Côte d'Ivoire. Sciences \& Nature, 7(1): 3134.

DOI: http://dx.doi.org/10.4314/scinat.v7i1.599 18

Pernezny KL, Roberts PD, Murphy JF, Goldberg NP. 2003. Compendium of Pepper Diseases. American Phytopathology Society Press, St Paul, $\mathrm{MN}$.

Potts MJ, Gunadi N. 1991. The influence of intercropping with Allium on some 
insect populations in potato (Solanum tuberosum). Annals of Applied Biology, 119: $297-213$.

R Core Team. 2017. R: A language and environment for statistical computing. $\mathrm{R}$ Foundation for Statistical Computing, Vienna, Austria. URL https://www.Rproject.org

Ryu JG, Ko SJ, Lee YH, Kim MK, Kim KH, Kim HT, Choi HS. 2009. Incidence and distribution of virus diseases on paprika (Capsicum annuum var. grossum) in jeonnam province of Korea. The Plant Pathology Journal, 25: 95-98.

Schiffers B, Mar A. 2011. Sécurité des opérateurs et bonnes pratiques phytosanitaires. Manuel $\mathrm{n}^{\circ} \quad 4$ : COLEACP/PIP Press. Bruxelles / Belgique : Programme PIP/COLEACP. $246 \mathrm{p}$.

Son D, Somda I, Legreve A, Schiffers B. 2017. Pratiques phytosanitaires des producteurs de tomates du Burkina Faso et risques pour la santé et l'environnement. Cahier Agriculture, 26: 25005.

DOI:

http://hdl.handle.net/2268/212922

Tossounon GAR, Onzo A. 2015. Inventaire des acariens et insectes ravageurs associés à la culture du piment vert Capsicum chinense Jacq. (Solanales: Solanaceae) dans les communes de Kandi et Malanville au Nord-Bénin. Annale de l'Université de Parakou, Série Science. Naturelles et Agronomie, 5: 111.

Zimmer AR, Leonardi B, Miron D, Schapoval E, Oliveira JR, Gosmann G. 2012. Antioxidant and anti-inflammatory properties of Capsicum baccatum: From traditional use to scientific approach. Journal of Ethnopharmacology, 139(1): 228-233.

DOI: 